Int. J. Mol. Sci. 2002, 3, 550-569

International Journal of

Molecular Science

ISSN 1422-0067

www.mdpi.org/ijms/

\title{
Use of a New Cluster Ansatz to Treat Strong Relaxation and Correlation Effects: A Direct Method for Energy Differences
}

\author{
Debasis Jana, Uttam Sinha Mahapatra and Debashis Mukherjee ${ }^{\dagger \star}$ \\ Department of Physical Chemistry, Indian Association for the Cultivation of Science, \\ Calcutta 700 032, INDIA. Tel.: +91-33-473 4688 Fax.: +91-33-483 6561 \\ ${ }^{\dagger}$ Also at Jawaharlal Nehru Centre for Advanced Scientific Research, Bangalore 560 064, INDIA. \\ *Author for correspondence. E-mail: pcdm@mahendra.iacs.res.in
}

Received: 11 January 2002 / Accepted: 3 February 2002 / Published: 31 May 2002

\begin{abstract}
We have presented in this paper a new cluster Ansatz for the wave operator for open-shell and/or quasidegenerate states, which takes care of strong relaxation and correlation effects in a compact and efficient manner. This Ansatz allows contraction among the various cluster operators via spectator orbitals, accompanied by suitable combinatorial factors. Since both the orbital and the correlation relaxations are treated on the same footing, it allows us to develop a very useful direct method for energy differences for open shell states relative to a closed-shell ground state, where the total charge for the two states may differ. We have discussed a new spin-free coupled cluster (CC) based direct method and illustrated its performance by evaluating electron affinity of a neutral doublet radical. We have also indicated how the scope of the theory can be extended to compute the state energies of simple open shell configurations as well. In that case, the CC equations terminate after the quartic power of cluster operators - exactly as in the closed-shell situation, which is not the case for the current methods.
\end{abstract}

Keywords: Valence-universal; valence-specific; strong relaxation and correlation effects; spin-free; coupled cluster; wave operator.

(C)2002 by MDPI, Basel, Switzerland. Reproduction for noncommercial purposes permitted. 


\section{Introduction}

Inspired by the pre-eminent success of the single reference coupled cluster (SRCC) theory for closed-shells [1-3] , several methodologies have been put forward for open-shell states which are either of single configuration type or which possess pronounced multi-reference (MR) character [4-12]. For the CC theory based on simple open-shell configurations (OSCC), the most common choice for the wave operator has been a simple exponential [13-18] exactly analogous to what is used in the SRCC theory. The more general MRCC developments, however, are not necessarily based on the use of a single exponential, and alternative forms of the wave operator based on normal ordered exponentials or using multiple exponentials have been proposed. Two general approaches have been followed for the MRCC developments. One of them is the valence-universal (VU) method $[4,9]$, tailored to treat energy differences of states with different degrees of ionization relative to a closed-shell ground state [4-12]. While the method of [4, 5] used ordinary exponential, the later theories generally use a normal ordered exponential [6-8,12]. These are explicitly spinfree, but require hierarchical generation of cluster amplitudes of various valence ranks. The other approach, leading to the valence-specific (VS) methods [10, 11], generate state-energies per se directly - without solving a hierarchy. They use an Ansatz where different exponentials acting on different model space functions. In contrast to the VUCC methods, the cluster amplitudes in these methods have to be generally defined in terms of spin-orbitals. This leads to spin-broken solutions [12] for the non-singlet states. This difficulty also showed up in OSCC theories which used only excitation operators without involving the spectators. In this case also, one has to necessarily use the spin-orbitals in defining the cluster operators [13-15]. An advantage however, of this Ansatz is that it retains the compactness and simplicity of the closed-shell SRCC method in generating $\mathrm{CC}$ equations which are at most quartic in cluster amplitudes.

A way out of the spin-contamination problem is to use a symmetry-adapted CC expansion, but the spin-adapted expressions will be unwieldy [19]. A partial resolution of the spin-contamination problem is to employ constraints on the expectation value of the $S^{2}$ operator [20]. The first explicitly spin-free OSCC method was suggested by Janssen and Schaefer [16]. They emphasized that, to maintain exact spin symmetry and to span the full spin-space, one would have to use spin-free operators in the cluster Ansatz and, would generally have to include excitation operators involving spectator scatterings of the electrons occupying active orbitals. This feature has since been explored in the later spin-free developments of OSCC theory [17, 18], where simple openshell singlets, doublets, and triplets have been treated on the same footing. All these theories make explicit use of spin-free generators (generically denoted for now on by the symbols $E_{i}^{j}$ with orbital labels $i$ and $j$ ) in the representation of $H$ and $\Omega$. For a general discussion of the spin-free formulation of the MRCC methods, we refer to Ref.[21]. 
All the formalisms using operators with spectators in an exponential representation for $\Omega$ have the disadvantage of using non-commuting cluster operators, leading to rather complex set of CC equations, containing up to octic powers of cluster amplitudes for single and double excitations [1618]. To avoid this complication, another spin-free formulation, was suggested from our laboratory [22]. This uses a cluster Ansatz which is neither a simple exponential as in [4,5], or the more widely used normal ordered exponential [6-8,12] for open-shells. This Ansatz allows for contractions among cluster operators via the spectator orbitals. The combinatoric factors associated with a composite obtained from such contractions involving $n$ cluster operators are not usually $(n !)^{-1}$, but depends on the number of ways these operators can be contracted using spectators, where these cluster operators appear contracted among themselves in all possible order. As is well-known, one can generate methods for energy differences relative to the energy $E_{0}$ of the closed-shell ground state (taken as the vacuum) using a factorization Ansatz involving wave-operator components $\Omega_{C}$ and $\Omega_{V}$ for core and valence respectively, from the Bloch equation involving the transformed hamiltonian $\bar{H}=\left(\Omega_{C}^{-1} H \Omega_{C}-E_{0}\right)$, with $\Omega_{C}=\exp (T)$ where $T$ is the cluster operator of the ground state. This idea was explored in Ref. [22] to generate a set of compact spin-free CC equations for electron detachment energies with respect to a closed-shell reference state. The preliminary treatment described in [22] demonstrated that the Ansatz leads to CC equations which are much less unwieldy as compared with the formulations described in [16-18]. It was also demonstrated that the method leads to very good IP values for core ionizations, which is dominated by large orbital relaxation effects.

Recently we have formulated a general version of the above method where the compactness of the CC equations was explicitly shown by working out the formal structure of the resulting CC equations for energy differences involving states of either simple open-shell nature or of arbitrary complexity. A succinct account of the formulation is about to appear in [23]. In the present paper, we discuss the method in some detail, and illustrate its performance by computing the electron affinity of $\mathrm{OH}$ radical, viewed as an IP of the $\mathrm{OH}^{-}$anion at the radical geometry.

\section{The New Ansatz for the Wave-operator and the Formulation of the CC Theory for Energy Differences}

\subsection{Preliminaries}

In this section we shall present the essential ingredients of the new formalism and show the underlying simplification brought out by our choice of the new wave operator. We shall illustrate the formalism for simple open-shell configurations.

Using the terminology of the effective hamiltonian formalism, we denote the projector onto the 
model space by $P$ and the one for the virtual space by $Q$. The Bloch equation for $\Omega$ is given by

$$
H \Omega P=\Omega P H \Omega P
$$

using the 'intermediate normalization' convention $P \Omega P=P$. The various components of $\Omega$ are obtained from the set of equations

$$
Q H \Omega P=Q \Omega P H \Omega P
$$

and $H_{\text {eff }}$ is given by

$$
H_{e f f}=P H \Omega P
$$

If instead of $E$, we are interested in getting energy differences $\Delta E$ relative to a closed-shell ground state $E_{0}$, then we posit the following factorized Ansatz [4, 5, 24]

$$
\Omega=\Omega_{C} \Omega_{V}
$$

where $\Omega_{C}$ is the wave-operator for the ground state $\Psi_{0}$, and $\Omega_{V}$ is the open-shell (or the 'Valence') component of $\Omega$ for the state of interest $\Psi$ :

$$
\begin{aligned}
\Psi_{0} & =\Omega_{C} \Phi_{0} \\
\Psi & =\Omega \Phi
\end{aligned}
$$

Introducing the transformed hamiltonian, $\bar{H}=\left(\Omega_{C}^{-1} H \Omega_{C}-E_{0}\right)$, we obtain

$$
\bar{H} \Omega_{V} P=\Omega_{V} P \bar{H} \Omega_{V} P
$$

and

$$
P \bar{H} \Omega_{V} P \equiv \bar{H}_{e f f}
$$

where the energy differences $\Delta E$ are obtained by diagonalizing $\overline{H_{\text {eff }}}$. Since $\Omega_{V}$ has valence destruction operators, $\Omega_{V} \Phi_{0}=0$, it then follows that the same $\Omega$ generates from $\Phi_{0}$ and $\Phi$ the corresponding eigenstates $\Psi_{0}$ and $\Psi$. This method for energy differences therefore belongs to VU category $[4,5,9]$.

In our applications in this paper, the space of $P$ is spanned by a single spin-adapted reference configuration $\Phi$, which is not necessarily a single determinant. It then follows that

$$
E=H_{e f f}=\langle\Phi|H \Omega| \Phi\rangle
$$

and

$$
\Delta E=E-H_{0}=\bar{H}_{\text {eff }}=\left\langle\Phi\left|\bar{H} \Omega_{V}\right| \Phi\right\rangle
$$

If we confine ourselves to just the valence sector of Fock space spanned by $\Phi$ and its virtual complements, then the equations above refer to a specific Hilbert space sector, and is a VS theory. 


\subsection{Normal ordered $V_{s}$ the New Ansatz for $\Omega_{V}$ and $\Omega$}

In the CC formulation, $\Omega_{C}=\exp (T)$, and $\Omega_{V}$ has to be chosen properly to get an extensive expression for $\Delta E$. We will, from now on, use a closed-shell function $\Phi_{0}$ as the vacuum to formulate our theory. In $\Omega_{V}$, or in the full $\Omega$ for open-shell energies, there will be additional cluster operators which we generically denote by $S$. We denote the holes by Greek letters $\alpha, \beta, \gamma$ etc, and the particles by Latin letters $p, q, r$, etc.

For the theories for energy-differences, rather than for state-energies per se, it is important to develop models of differential correlation. In such situations, CC theories treat the closedshell ground state in terms of various $\mathrm{n}$ hole $-\mathrm{n}$ particle $(n h-n p)$ cluster operators, and the excited/ionized states of interest are described by a cluster-expanded wave-operator which include - in addition to those pertaining to the ground state - extra valence cluster operators $S_{e}$ involving excitations into or out of the partially filled 'valence' or 'active' orbitals (specially those inducing open-shell correlation effects), and operators $S_{r}$ which bring in differential correlation and orbital relaxation effects. These latter cluster operators involve again the various $\mathrm{n}$ hole $-\mathrm{n}$ particle excitations, but additionally have excitations out of or into valence orbitals. These are thus valence cluster operators. An important class of valence cluster operators involves $\mathrm{n}$ hole $-\mathrm{n}$ particle excitations in the presence of a passive scattering of electrons between the same valence orbitals. They are cluster operators with spectator valence or active lines. The overall effective $n h-n p$ excitation amplitudes from an open-shell configuration is thus a sum of the parent groundstate $n h-n p$ amplitude and the additional amplitudes containing spectator valence orbitals. The latter ones thus bring in the relaxation of the ground state excitation amplitudes to the values they should have in the open-shell configurations, and thus bring in the differential correlation/orbital relaxation effects. In case one starts out with the set of mean-field orbitals that are optimal for the closed-shell ground state (i.e. the ground state Hartree-Fock (HF) orbitals), the $1 h-1 p$ excitations with spectator orbitals bring in orbital relaxation effects and $2 h-2 p$ excitations with spectator orbitals bring in the dominant differential correlation effects.

The traditional CC based correlation theories for energy differences posit on the $\Omega_{V}$ a normal ordered exponential [4-9,12] involving the open-shell excitation operators $S_{e}$ and the relaxation/differential correlation operators $S_{r}$. The normal ordering in $\Omega_{V} \sim\left\{\exp \left(S_{e}+S_{r}\right)\right\}$ is performed with respect to $\Phi_{0}$ taken as vacuum. The valence-universality of $\Omega_{V}$ implies that $\Omega_{V}$ is the same for all the model spaces $\mathcal{S}_{m}$ where m runs from $N_{V}$, the target $N_{V}$ - valence space, all the way down to 1 , the one-valence space. Owing to the normal ordering in $\Omega_{V}$, there is a hierarchical decoupling of the cluster-amplitudes $S_{e}$ and $S_{r}$ of different valence ranks [9]. The use of the closedshell $\Phi_{0}$ as the vacuum ensures that $S_{e}$ and $S_{r}$ are spin-scalars and can be described by spin-free unitary generators [16-18]. This makes the spin-adaptation a rather simple and straightforward exercise. 
The advantages of the normal ordered Ansatz for $\Omega$ are, however, off-set somewhat by two difficulties. One is that the use of a valence-universal $\Omega_{V}$ implies solving for the cluster-amplitudes of $S_{e}(m)$ and $S_{r}(m)$ of all valence ranks $1 \leq m \leq N_{V}$, even if we are ultimately interested in the target $N_{V}$-valence situation. This is an unnecessary exercise. The other difficulty is physically more interesting, and throws light on the limitation of a normal ordered Ansatz for $\Omega_{V}$ to tackle relaxation and differential correlation effects. The normal ordering in $\Omega_{V}$ prevents contractions between all the $S$ operators. As a result, the powers $S^{k}$ from $\Omega_{V}$ involving valence excitations with more than $\mathrm{m}$ orbitals for an m-valence model space $\mathcal{S}_{m}$ automatically gives zero. However, the various $n h-n p$ cluster operator $T_{n}$ have all powers active in $\Omega_{C}$ (with $1 \leq n \leq N_{C}$, with $N_{C}$ electrons in $\Phi_{0}$ ); so that $n h-n p S_{r}$ s should be present in the same powers. If we denote by ${ }^{(n)} S_{r}^{(l)}$ an arbitrary $n h-n p$ excitation with $l$ spectator orbitals, then it is physically reasonable to demand that the effective $n h-n p$ excitation operators in $\Omega_{V}$ for the open-shell situation for the m-valence $\mathcal{S}_{m}$ should contain all the powers of each of ${ }^{(n)} S_{r}^{(l)}(1 \leq l \leq m)$ should be present. This is, however, precluded by the very nature of the normal ordered $\Omega_{V}$. For a one-valence problem as in the IP calculations, the amplitudes such as $\left(s_{r}\right)_{\gamma \alpha}^{p \alpha}$ and $\left(s_{r}\right)_{\gamma \delta \alpha}^{p q \alpha}$ bring in orbital relaxation and two-body correlation relaxation effects, respectively; $\alpha$ is the valence hole label for a one-valence open-shell model function $\Phi_{\alpha}$. Since $\Omega_{C}$ generates all powers of $T_{1}$ and $T_{2}$, acting on $\Phi_{\alpha}$, with $T_{1} \equiv \sum_{p \gamma} t_{\gamma}^{p} E_{\gamma}^{p}$ and $T_{2} \equiv \frac{1}{2} \sum_{p q \gamma \delta} t_{\gamma \delta}^{p q} E_{\gamma \delta}^{p q}$, we need all powers of $\left(s_{r}\right)_{\gamma \alpha}^{p \alpha}$ and $\left(s_{r}\right)_{\gamma \delta \alpha}^{p q \alpha}$ amplitudes coming from $\Omega_{V}$ to fully take care of the orbital and pair correlation relaxation terms. However any power of $\left\{S_{r}^{k}\right\}$ with $k>1$ will involve destruction of more than one valence occupancy and will thus give zero by their action on $\Phi_{\alpha}$. As a result, $\Omega_{V} \Phi_{\alpha}$ is effectively just $\left\{1+S_{r}^{(1)}\right\} \Phi_{\alpha}$, and misses the powers of $S_{r}^{(1)}$ which are crucial when the relaxation or the differential correlations effects are large. For the core-IP, the orbital relaxation of the neutral HF orbitals is very large [25] and the usual normal ordered exponential based VUCC methods would fail in a significant manner.

There is an earlier MRCC formulation by Mukhopadhyay and Mukherjee [26, 27] which treats the orbital relaxations and the correlation relaxations on the same footing as in the ground state by invoking the Jeziorski and Monkhorst (JM) type of Ansatz for $\Omega_{V}[10,11]$ advocated for their valence-specific MRCC (VS-MRCC) theories for state-energies per se. The modification consists in merely replacing the microscopic hamiltonian $H$ by the dressed hamiltonian $\bar{H}=$ $\exp (-T) H \exp (T)-E_{\mathrm{gr}}$, with $E_{\mathrm{gr}}$ as the exact ground state energy. This so called quasi-Fock MRCC is then a method for computing energy differences. $\Omega_{V}$ is written as $\Omega_{V}=\sum_{\mu} \exp \left(S^{\mu}\right)\left|\Phi_{\mu}\right\rangle$ $\left\langle\Phi_{\mu}\right|$, as in JM formulation and each $S^{\mu}$ involves all $n$-body excitations from each $\Phi_{\mu}$ which are themselves taken as vacuum; there are thus no spectator labels. Since it is again the full exponential $\exp \left(S^{\mu}\right)$ which acts on each $\Phi_{\mu}$, the orbital relaxation and correlation effects are treated to all powers. There is, however, a big price to pay. Since in general, the functions $\Phi_{\mu}$ will be spin-nonsinglets (they will be doublet functions for IP calculations, for example), the operators 
$S^{\mu}$ cannot easily be chosen in a manifestly spin-free form. The use of spin-orbital based amplitudes in $S^{\mu}$ would not only proliferate the number of cluster amplitudes, they would also generally lead to spin-broken solutions. It is now well-documented that, even if $S^{\mu} \Phi_{\mu}$ is explicitly spin-adapted, the powers $\left(S^{\mu}\right)^{k} \Phi_{\mu}$ are not necessarily so. Thus, in practice, though the use of $\exp \left(S^{\mu}\right)$ solves the problem of limited inclusion of orbital and correlation relaxation effects as compared to that in VU-MRCC theories, the spin-orbital formulation and the consequent spin-contamination [12] is a major deterrent for the quasi-Fock MRCC theories using the JM-type formalism.

What is obviously warranted is the flexibility in $\Omega_{V}$ of the spin-free representation of the VUMRCC approach (which implies that only a singlet type vacuum has to be adopted), and at the same time allowing the exponentiation of the $S$ operators in contrast to a normal ordered $\Omega_{V}$. A preliminary formulation to achieve these twin goals was initiated some years ago by Mukhopadhyay et al [28]. In this method, the $S_{r}$ operators were allowed to contract with the spectator lines. There were several limitations of this formalism. The most important among them have been (a) the potential non-termination of the MRCC equations, since the $S_{r}$ operators could be joined in the equations in a chain-like fashion up to arbitrary powers [22, 23]: In contrast the normal ordered exponential $\Omega_{V}$ or the closed-shell $\Omega_{\mathrm{s}}$ lead only to a finite power of cluster amplitudes since all cluster operators have to be joined to $\mathrm{H}$; (b) there was no way of factorizing out the $S_{r}$ operators joined to other $S_{r}$ operators and not joined to $\mathrm{H}$ to lead to a more compact form of the MRCC equations. This last aspect was a direct consequence of the choice of the $\Omega_{V}$ in [28] which allowed powers of $S_{r}$, but not with the proper factors which would have offered the factorization.

The relaxation-inducing cluster expansion formalism for $\Omega_{V}$ we are going to discuss in this paper gets rid of the above limitations by postulating an Ansatz for the $\Omega_{V}$ which allows restricted contractions between the $S$ operators and affixes specific combinatoric factors with each such powers of contracted $S$ operators. The specific choice of these combinatoric factors is very crucial for us, since this leads to the generation of finite power series in cluster amplitudes for the associated MRCC equations. The theory is very general with respect to the number of valence (active) electrons or holes present in the model spaces. In this paper, however, we should discuss explicitly only the one-valence case. We use a suitable closed-shell vacuum for defining our $\Omega_{C}$ and $\Omega_{V}$. This leads to a manifestly spin-free form for the cluster operators $S$ in $\Omega_{V}$. A straightforward use of this $\Omega_{V}$ in the Bloch equation for energy-differences leads to a potentially non-terminating series of $S$ operators in the MRCC equations, somewhat similar to what was obtained by Mukhopadhyay et al [28]. However, we show that the use of the specific combinatoric factors for the powers of contracted $S$ in $\Omega_{V}$ leads to a set of equivalent MRCC equations where all the $S$ operators are connected directly to the dressed hamiltonian $\bar{H}$. This lends a finite power-series structure to the resultant MRCC equations. Preliminary versions of the formalism for the one-valence case has already been published [22]. A brief account of the general versions has been published already 
[23].

We motivate towards our development with the example of the one-valence problem. Our model function $\Phi_{\alpha}=a_{\alpha} \Phi_{0}$ is a doublet. Spectator scatterings must have to be generally included in the spin-free choice of the cluster operators to exhaust the configurations in the $Q$ space which have the same orbitals but which differ in the spin functions. Thus, to incorporate the linearly independent single excitations from a hole $\gamma$ to a particle $p$, we need two linearly independent amplitudes, corresponding essentially to excitations with up and down spins for orbitals $\gamma$ and $p$. This can be realized by choosing the two excitation operators as $\left\{E_{\gamma \alpha}^{p \alpha}\right\}$ and $\left\{E_{\alpha \gamma}^{p \alpha}\right\}$. The curly braces denotes normal ordering with respect to closed-shell $\Phi_{0}$. In these operators there is a spectator scattering involving the active orbital $\alpha$ in the direct and exchange modes respectively.

Another possible choice for two linearly independent excitations could have been $\left\{E_{\gamma}^{p}\right\}$ and $\left\{E_{\alpha \gamma}^{p \alpha}\right\}$. In the present formulation we would prefer to keep the spectator scattering in the direct term, so will use $\left\{E_{\alpha \gamma}^{p \alpha}\right\}$. This choice is more convenient for treating theories for energies $E$ and $\Delta E$ on the same footing. The single excitation like $s_{\gamma}^{\alpha}\left\{E_{\gamma}^{\alpha}\right\}$ is of the type $S_{e}$, while operators like $s_{\gamma \alpha}^{p \alpha}\left\{E_{\gamma \alpha}^{p \alpha}\right\}$ and $s_{\alpha \gamma}^{p \alpha}\left\{E_{\alpha \gamma}^{p \alpha}\right\}$ are of the $S_{r}$ type. Since, in the theory of the energy differences, the overall single excitation amplitude for the excitation $\gamma \rightarrow p$ will be dictated by suitable combination of the closed-shell amplitude $t_{\gamma}^{p}$ and the valence amplitudes $s_{\gamma \alpha}^{p \alpha}$ and $s_{\alpha \gamma}^{p \alpha}$, coming from $\Omega \equiv \Omega_{C} \Omega_{V}$ the effect of the $s$ amplitudes is to 'relax' the value of the closed-shell amplitude $t_{\gamma}^{p}$ to the value appropriate for the doublet states. This is why we label the part of $S$ operators containing spectators by the symbol $S_{r}$.

As we have emphasized, the traditional normal ordered cluster Ansatz $\Omega_{V} \equiv\{\exp (S)\}[6,7,9]$ does not use the full power of the exponential structure, when acting on the reference function $\Phi$, owing to its normal ordered form. For the doublet function $\Phi_{\alpha},\{\exp (S)\}$ acting on $\Phi_{\alpha}$ is essentially $\{1+S\} \Phi_{\alpha}$. The operators $\left\{E_{\gamma \alpha}^{p \alpha}\right\}$ and $\left\{E_{\alpha \gamma}^{p \alpha}\right\}$ are just single excitations, which modify the orbitals. If we start out with the orbitals for the neutral vacuum state $\Phi_{0}$, these are not the best choice for the cation described by $\Omega \Phi_{\alpha}$. However, presence of all powers of single excitations - as in an exponential - would have taken care of the orbital relaxation via the so-called Thouless Theorem [29]. The normal ordered form $\{1+S\} \Phi_{\alpha}$ cannot provide such powers of excitations. Although a simple exponential choice $\Omega_{V}=\exp (S)[4,5]$, will provide exponentiation of $S$ via the powers, the factors $(n !)^{-1}$ coming from the exponential are not the proper combinatoric factors (for setting a compact power series expansion in the CC equations) when $S_{e}$ operators are contracted to $S_{r}$ via spectator orbitals. The correct combinatoric factors can be ascertained on physical grounds. Each term in $\Omega$ (or $\Omega_{V}$ ) must lead to multiple excitations via product of cluster operators such that each distinct product excitation should appear only once with a factor 1 . In case contractions between $S$ operators are permitted, the factor $(n !)^{-1}$ imply that all the $n S$ operators can be joined among themselves in all possible $n$ ! ways. But it may not be possible for all $n S$ operators to be joined 
in such a way as to lead to same product excitation.

We want to have an Ansatz for $\Omega$ which allows contractions between operators viz. spectators, and at the same time demand that each term of the various product excitations - appearing either uncontracted to one another under the normal order or appearing as composites after contractions-should appear only once, as in the ordinary exponential without spectators. It then follows that we want to have a series for $\Omega$ in normal order where the various composites, $n$ in number, which appear uncontracted under the normal order should appear with a factor $(n !)^{-1}$, corresponding to $(n !)$ various different ways the composites can appear. However in case there are contractions between $n$ operators, but only there are $f_{n}$ ways of joining them, then we should attach a weight $f_{n}^{-1}$ to the composite to ensure that the various ways of joining the $S$ operators in the composite leading to the same product excitations should appear only once. We note here that the commuting operators in $\exp (T)$ realizes this automatically in the SRCC theory for closedshells. If the operators do not commute, as for $S_{e}$ and $S_{r}$, where $S_{e}$ has no spectators and $S_{r}$ has spectators, the ordinary exponential introduces unphysical weights such as $(2 !)^{-1}$ for quadratic powers for the contracted composites and so on. But we can have only a composite $\overline{\left\{S_{e} S_{r}\right\}}$ and not $\overline{\left\{S_{r} S_{e}\right\}}$, and hence $\overline{\left\{S_{e} S_{r}\right\}}$ should appear with a factor 1 .

We illustrate this with a concrete example with $\phi_{\alpha}$ as the model function. All the possible $S$ operators are shown in Fig. 1 Let us consider the product excitations coming from an $S_{e}$ as $s_{\gamma}^{\alpha}\left\{E_{\gamma}^{\alpha}\right\}$ and $S_{r}$ as $s_{\gamma \alpha}^{p \alpha}\left\{E_{\gamma \alpha}^{p \alpha}\right\}$. If we use a pure exponential for $\Omega_{V}$, then the product excitation leading to double excitation $\gamma \delta \rightarrow \alpha p$ would appear with a factor $(2 !)^{-1}$. The operators $s_{\gamma}^{\alpha}\left\{E_{\gamma}^{\alpha}\right\}$ can contract with $s_{\gamma \alpha}^{p \alpha}\left\{E_{\gamma \alpha}^{p \alpha}\right\}$ only from the left via $\alpha$. But put in the reversed order, they cannot be contracted from the right. If we want to have each distinct type of product excitation to appear only once, the factor with the composite obtained by joining the operators $s_{\gamma}^{\alpha}\left\{E_{\gamma}^{\alpha}\right\}$ and $s_{\gamma \alpha}^{p \alpha}\left\{E_{\gamma \alpha}^{p \alpha}\right\}$ should just be 1.

In general then, the correct combinatoric factors would appear if we assign a factor $f^{-1}$ to a composite obtained by contracting $k S_{e}$ operators to $l S_{r}$ operators, where $f$ is the number of possible ways of joining them together leading to composites of same excitation. With this insight, the contracted product excitation from $s_{\gamma}^{\alpha}\left\{E_{\gamma}^{\alpha}\right\}$ and $s_{\gamma \alpha}^{p \alpha}\left\{E_{\gamma \alpha}^{p \alpha}\right\}$ would have a weight of 1. Clearly no $S_{e}$ operators can be joined to $S_{r}$ operators from right, and all $S_{r}$ operators need not all be joined among themselves to form the composite.

To take care of the proper factors in the composites obtained via spectator contractions, we have thus suggested recently [22] that $\Omega_{V}$ should be taken to be of a combinatoric cluster expansion form:

$$
\Omega_{V}=\{\{\exp (S)\}\} \equiv\left\{\left\{\exp \left(S_{e}+S_{r}\right)\right\}\right\}
$$

where $\{\{\cdots\}\}$ denotes a special ordering. It allows contraction of the $S$ operators via spectator lines, but it assigns the appropriate combinatoric factors $f^{-1}$ to each composite. 


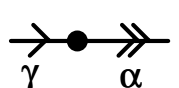

(a)

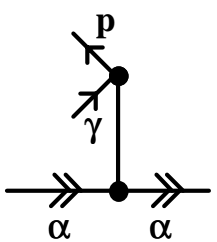

(c)

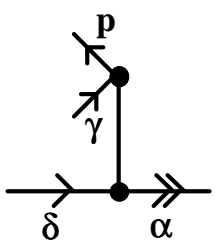

(b)

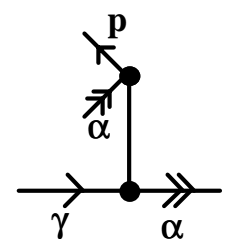

(d)

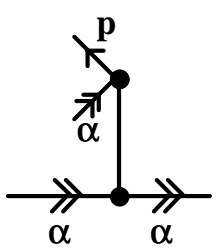

(e )

Figure 1: The various types of $S$ operators for the one-hole model space $\Phi_{\alpha}$. (a) and (b) are $S_{e}$ operators, and the rest are $S_{r}$.

From now on, we shall call all composites leading to the same excitation, but having different ordering of connectivities via spectators, as topologically equivalent. All the equivalent composites have the same 'topological weight', $f^{-1}$. Their overall contribution to the excitation can thus be taken care of by considering only one of them with a factor of 1 . It turns out that the classification of the various terms in the open-shell CC equations are best done in terms of composites of equivalent topologies.

\subsection{Emergence of Strongly Connected Finite Power MRCC Equations}

Let us now rewrite the left side of the eq. (7) in normal order with respect to $\Phi_{0}$, using the Ansatz eq. (11) for $\Omega_{V}$. It is straightforward to show that the resultant terms in normal order can be written as

$$
\bar{H} \Omega_{V}=\bar{H}\{\{\exp (S)\}\}=\left\{[\overline{\bar{H}\{\{\exp (S)\}\}}] \Omega_{V}\right\}
$$

where the connected composite $\overline{\bar{H}\{\{\exp (S)\}\}}$ is obtained by joining $\bar{H}$ with various powers of $S$ in all possible ways, at the same time joining $S_{e}$ and $S_{r}$ operators among themselves in all possible 
ways. The factors associated with the composites are according to the definition of $\{\{\exp (S)\}\}$. The various terms of all powers of $S$ not joined to the composites can all be grouped again to form $\Omega_{V}$. Using eq. (8), the right side of eq. (7) can be written as

$$
\Omega_{V} P \bar{H} \Omega_{V} P \equiv \Omega_{V} \bar{H}_{e f f} P \equiv\left\{\Omega_{V}\left[\overline{\{\{\exp (S)\}\} \bar{H}_{e f f}}\right]\right\}
$$

where $\left[\overline{\{\{\exp (S)\}\} \bar{H}_{e f f}}\right]$ is the composite obtained by joining powers of $S$ with $\bar{H}_{\text {eff }}$ in all possible ways, at the same time appropriately joining $S_{e}$ and $S_{r}$ among themselves. Using the linear independence of all the operators of $\Omega_{V}$ in a $\mathrm{VU}$ theory $[4,5,9]$, it then follows that

$$
\{[\overline{\bar{H}}\{\{\exp (S)\}\}]\}=\left\{\left[\overline{\{\{\exp (S)\}\} \bar{H}_{\text {eff }}}\right]\right\}
$$

Since all powers of $S$ lead to excitations out of the $P$ space, the closed (or the model space projection) components of both sides of the equations lead to

$$
\{[\overline{\bar{H}}\{\{\exp (S)\}\}]\}_{c l}=\bar{H}_{e f f}
$$

Inspection of the left side of eq. (15) reveals very specific modes of connectivity of the various composites appearing in it. Any $S$ operator in a composite joined either to another $S$ operator via the spectator lines only, or to the $\bar{H}$ via the spectator lines only must leave some of its inactive orbitals uncontracted, and hence cannot contribute to the closed projection. We call such type of connectivity as 'weak connectivity' $[22,23]$. The rest of the terms would have $S$ operators joined to $\bar{H}$ by at least one inactive line and, in addition may generally have contractions among themselves via spectator lines. We call these composites 'strongly connected' $[22,23]$. The above argument shows that $\bar{H}_{\text {eff }}$ is strongly connected, and thus cannot have more than the powers of $S$ exceeding number of lines in $\bar{H}$.

We now regroup the various terms of eq. (14) in terms of strongly connected entities. Let us consider the left side of eq. (14) first. Any general term of the left side will have several $S$ operators joined strongly to $\bar{H}$ (i.e. not just by spectator lines), in addition to connection among themselves via the spectator lines, and additionally we have other $S$ operators joined just to other $S$ operators (or to $\bar{H}$ ) via the spectator lines. These latter are thus weakly connected. Several composites will have the same strongly connected terms, but they will differ in the ways the $S$ operators are joined weakly to them. We denote the various strongly connected components by $X_{i}$, where $i$ distinguishes the various terms. All the composites with the same strongly connected component and the same $S$ operators joined weakly to this components in various ways leading to same shape may be termed as weakly connected composites of same topology. Each such term will have some $S_{e}$ operators joined weakly to $S_{r}$ operators via spectator lines. The $S_{r}$ operators to which they are connected are either a part of $X_{i}$ 's (viz. they are strongly connected to $\bar{H}$ ), or 


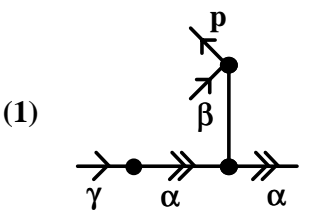

( a )

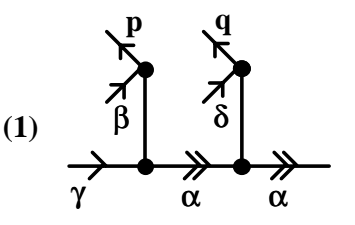

(b)

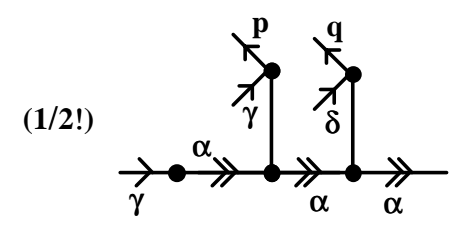

(c)

Figure 2: Connectivities of $S_{e}$ and $S_{r}$ operators, having different factors: (a) one-body $S_{e}$ and $S_{r}$, (b) two-body $S_{e}$ and $S_{r}$ and (c) one-body $S_{e}$ and two $S_{r}$ operators. The weight factors are indicated in the parentheses.

they themselves are weakly connected to $X_{i}$ 's. It is interesting that the contributions of all the weakly connected composites of same topology can be written as coming from just one term in which each weakly connected $S_{e}$ operator is joined to $S_{r}$ operators which are strongly connected to $\bar{H}$, i.e. they, a part of $X_{i}$ 's, and all the weakly connected $S_{r}$ operators are joined from the right to $X_{i}$ 's via spectator lines only. The weight of this term is $f^{-1}$ where $f$ is the number of ways the various weakly connected $S_{e}$ and $S_{r}$ operators can be arranged among themselves. This is shown in Fig. 2. The entire term on the left side of eq. (14) can then be written as

$$
\{[\bar{H}\{\{\exp (S)\}\}]\}=\left\{\overline{\left[\left\{\exp \left(S_{e}\right)\right\}\right]_{w} X\left[\left\{\left\{\exp \left(S_{r}\right)\right\}\right\}\right]_{w}}\right\}
$$

where each $S_{e}$ operator is weakly connected to $X$ via $S_{r}$ operators in $X$, and they are not joined to each other. We have made the convention of stretching them to the left of $X$ without any change in their contribution. This stretching is also depicted in Fig. 3(a). The two weakly connected $S_{r}$ operators appear only on the right of $X .[\cdots]_{w}$ denotes a term joined weakly to the rest of a composite. 


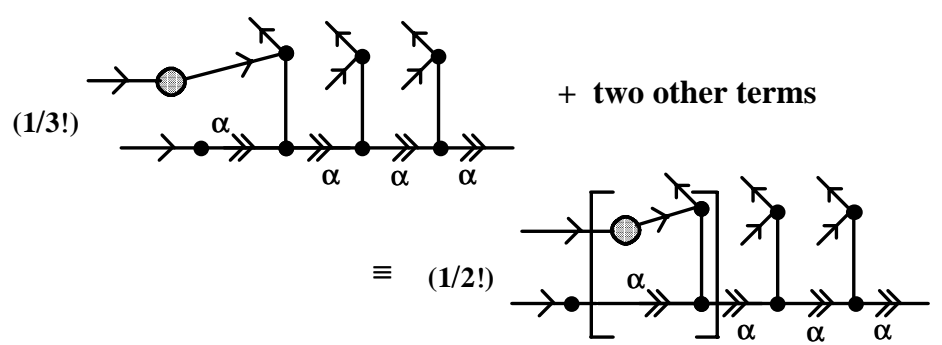

( a )

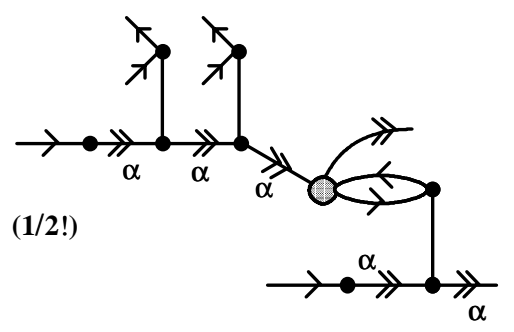

$(\mathbf{1} / \mathbf{2}$ !)

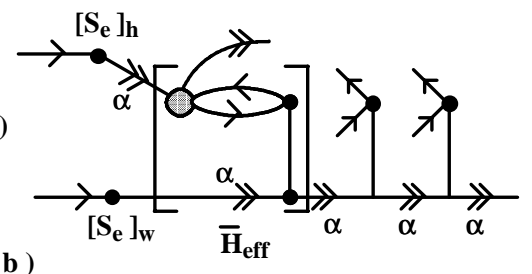

Figure 3: (a) Overall contribution from three diagrams of the same topology. The skeleton in the braces is the strongly connected ' $X$ ' operator. (b) Moving the weakly connected $S_{r}$ operator on the left of $\bar{H}_{\text {eff }}$ to its right, yielding the same contribution. The quantity in the braces is the $\bar{H}_{\text {eff }}$.

Using entirely the same reasoning on the right side of eq. (14), we have

$$
\left\{\left[\overline{\{\{\exp (S)\}\} \bar{H}_{e f f}}\right]\right\}=\left\{\left[\left\{\exp \left(S_{e}\right)\right\}\right]_{w}\left[\left\{\exp \left(S_{e}\right)\right\}\right]_{h} \bar{H}_{\text {eff }}\left[\left\{\left\{\exp \left(S_{r}\right)\right\}\right\}\right]_{w}\right\}
$$

where each $S_{e}$ in $[\cdots]_{h}$ above is joined entirely to the $\bar{H}$ vertex, and each $S_{e}$ in $[\cdots]_{w}$ is joined to one or more $S_{r}$ vertex which are part of $\bar{H}_{e f f}$. The $S_{r}$ operators, originally connected weakly among themselves via spectator lines from the left of $\bar{H}_{\text {eff }}$ are all moved to its left. This operation keeps the contribution of these terms unchanged, when they belong to the same topology. As an example, we have shown in Fig. 3(b) one term from eq. (17) where the $S_{r}$ vertices are taken from the left to the right of the $\bar{H}_{\text {eff }}$ vertex.

Again since all powers of $S_{r}$ in $\left\{\left\{\exp \left(S_{r}\right)\right\}\right\}$ are linearly independent, it follows from eqs. (14), (16) and (17) that

$$
\left\{\left[\left\{\exp \left(S_{e}\right)\right\}\right]_{w} X\right\}=\left\{\left[\left\{\exp \left(S_{e}\right)\right\}\right]_{w}\left[\left\{\exp \left(S_{e}\right)\right\}\right]_{h} \bar{H}_{e f f}\right\}
$$


'Inverting' the 'exponentials' in eq. (18) leads to

$$
\left\{\left[\left\{\exp \left(-S_{e}\right)\right\}\right]_{h} X\right\}=\bar{H}_{e f f}
$$

where each term in $\left\{\exp \left(-S_{e}\right)\right\}$ is joined to the $X$ via its $\bar{H}$ vertex, and they are not joined among themselves. The strongly connected composite $X$ has one set of terms $Z$ which are 'external' or 'open' in the sense of inducing excitations to the virtual space from the model space and another set $W$ which is 'closed'. Only $W$ contributes to $\bar{H}_{\text {eff }}$. Denoting the external operators by the suffix 'ex', and the closed ones by 'cl', we have, from the $Q$ projection of eq. (19), the relation

$$
\left[\left\{\left[\left\{\exp \left(-S_{e}\right)\right\}\right]_{h}[Z+W]\right\}\right]_{e x}=0
$$

The $P$ projection leads to

$$
W=X_{c l}=\bar{H}_{e f f} \equiv \Delta E
$$

Eqs. (20) and (21) are respectively our stipulated CC equations the VU theory for the cluster amplitudes for $S$ and for $\bar{H}_{e f f}$ in the spin-free compact formulation. Each $m$ - valence component of eq. (20) should separately be equated to zero to generate the $S^{(m)}$ 's. Because of the nature of connectivity, in an $m$ - valence component of eq. (20) no $S^{(l)}$ with $l>m$ appears, as in a normal ordered formulation.

It is clear from our formulation that the expression on the left hand of eq. (20) is finite power series in $S$, and since all $S$ operators are strongly connected to the vertex of $\bar{H}$. We should emphasize again that the finite series emerges entirely due to our new Ansatz for $\Omega_{V}$ with suitable combinatoric weights.

\subsection{Illustrative Applications to a Simple Open-shell Doublet}

We will now discuss the essentials of the construction of the working equations, eq. (20) and eq. (21), by applying it to a one-valence problem, viz. the CC formulation based on the open-shell reference (N-1)-electron doublet $\Phi_{\alpha}$ introduced in Sec. 2. We also truncate the rank of the $T$ and $S$ operators at the two-body level. The $S$ operators in this truncation scheme have all been already shown in Fig. 1. The various sets of equation from eq. (14) can be diagrammatically constructed by first constructing the strongly connected composites $Z$ and $W$ from $\bar{H}, S_{e}$, and $S_{r}$ vertices, and then connecting them by the excitation operators $S_{e}$ from their left via the $\bar{H}$-vertex, omitting connection among the $S_{e}$ operators. The various CC equations for the operators shown in Fig. 1 can be compactly written as

$$
\begin{aligned}
Z_{\gamma}^{\alpha} & +\left[s_{e \gamma}^{\alpha}\right]_{h} W_{\alpha}^{\alpha}=0 \\
Z_{\gamma \beta}^{\alpha p} & +\left[s_{e \gamma}^{\alpha}\right]_{h} Z_{\alpha \beta}^{\alpha p}+\left[s_{e \beta}^{\alpha}\right]_{h} Z_{\gamma \alpha}^{\alpha p}
\end{aligned}
$$




$$
\begin{aligned}
& +\left[\left\{s_{e \beta}^{\alpha} s_{e \gamma}^{\alpha}\right\}\right]_{h} Z_{\alpha \alpha}^{\alpha p}-\left[s_{e \gamma \beta}^{\alpha p}\right]_{h} W_{\alpha}^{\alpha}=0 \\
Z_{\alpha \beta}^{\alpha p} & +\left[s_{e \beta}^{\alpha}\right]_{h} Z_{\alpha \alpha}^{\alpha p}=0 \\
Z_{\beta \alpha}^{\alpha p} & +\left[s_{e \beta}^{\alpha}\right]_{h} Z_{\alpha \alpha}^{\alpha p}=0 \\
Z_{\alpha \alpha}^{\alpha p} & =0
\end{aligned}
$$

We should mention here that the positive signs in $\left[s_{e \gamma}^{p}\right]_{h} Z_{\alpha \beta}^{\alpha p}$ originate from two $(-1)$ factors, one coming from $\left\{\exp \left(-S_{e}\right)\right\}$ of eq. (21), and the other coming from one internal hole line joining $S_{e}$ and $Z$. The ionization potential (IP) is given by $W_{\alpha}^{\alpha}$.

\section{A Compact Open-shell CC Theory for Simple Open-shell States: The Doublet Case}

In this section, we shall briefly touch upon the essential modifications necessary to convert the formulation for energy differences described in Sec. 2 to a VS open-shell spin-free CC formalism for state energies per se. We will not give the detailed general proof in this paper, but will just illustrate the modification with the example of the doublet states starting from a single reference doublet determinant $\Phi_{\alpha}$.

The exact wave-function $\Psi$ in our open-shell CC theory is given by

$$
\Psi=\{\{\exp (\bar{S})\}\} \Phi_{\alpha}
$$

where $\{\{\cdots\}\}$ again denotes the new combinatoric cluster expansion, and $\bar{S}$ contains valence excitation and relaxation operators of exactly the same types as depicted for $S$ in Figs. 1(a)-(e). We do not have now any ground state cluster operators $T$, since this is not the theory for energy differences. Using the same manipulations as have been indicated in Sec. 2, we may again get a set of strongly connected composites contributing to the equations for the cluster amplitudes. For a concrete discussion, let us call the composite $\left\{\left[\left\{\exp \left(-S_{e}\right)\right\}\right]_{h}[Z+W]\right\}$ as $G$. The additional terms that need be considered in the VS theory are the pure hamiltonian vertices of lower ranks that would contribute to the CC equations corresponding to the blocks with direct spectator scatterings. The blocks $Z$ and $W$ are now constructed entirely from $H, S_{e}$ and $S_{r}$ operators.

The additional terms that have to be added are, respectively, $\left(-f_{\beta}^{p}\right)$ and $\left(-f_{\alpha}^{p}\right)$ for the CC equations eq. (24) and eq. (26) corresponding to the direct spectator scatterings on top of actual excitations $(\beta \rightarrow p)$ and $(\alpha \rightarrow p)$ :

$$
\begin{aligned}
& G_{\alpha \beta}^{\alpha p}-f_{\beta}^{p}=0 \\
& G_{\alpha \alpha}^{\alpha p}-f_{\alpha}^{p}=0
\end{aligned}
$$




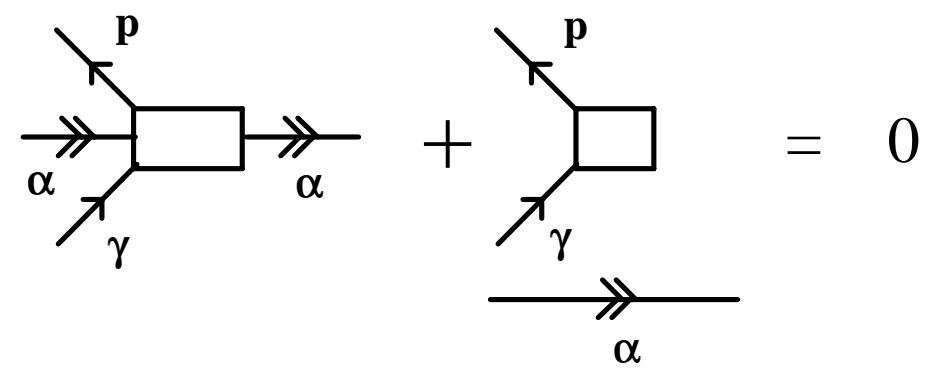

Block I

Block II

Figure 4: Block equation for pseudo-two-body excitations with spectator scattering line $\alpha . \gamma$ can take on labels $\beta$ and $\alpha$, leading to eqs. (28) and (29).

The corresponding block structure of the diagrams are shown in Fig. 4 The minus sign on the added terms is relative to these in eqs. (24) and (26) and is due to an 'extra' internal hole line in the diagrams of eqs. (24) and (26). All the $S_{r}$ operators in the modified CC equation for state energies are joined to the $H$ vertex, and the $S_{e}$ operators joined from the left are also joined to the $H$ vertex. The maximum power of all the $S$ operators in each term can thus be only quartic, exactly as in the closed-shell CC theory.

Some other considerations are warranted at this stage, if we confine ourselves to at most twobody cluster operators. Since we do not have $T$ operators in the VS formalism, the closedshell like analogue of two-hole two-particle excitations would not appear in our formalism at the two-body truncation level. However, these are the dominant type of correlations and, in the present formalism, would require three-body operators with direct spectators. The corresponding amplitude $s_{\gamma \delta \alpha}^{p q \alpha}$ would explicitly 'see' the presence of the spectator vacant orbital $\alpha$. The analogous equation would contain, in exact analogy with the single excitations $(\beta \rightarrow p)$ or $(\alpha \rightarrow p)$ in presence of spectators, as in eqs. (28) and (29), a composite containing three-body scattering $(\gamma \delta \alpha \rightarrow p q \alpha)$ with the direct spectator scattering involving $\alpha$, and another which would look like a pure two-body scattering $\gamma \delta \rightarrow p q$. This is illustrated diagrammatically in Fig. 5.

Written in terms of $G$, the relevant equation will look like

$$
G_{\gamma \delta \alpha}^{p q \alpha}-v_{\gamma \delta}^{p q}=0
$$

This is analogous to the modified equations (28) and (29) from eq. (24) and (26) for single excitations with spectator scattering. In case we do not want to include $s_{\gamma \delta \alpha}^{p q \alpha}$, as in our current application, we approximate it by $t_{\gamma \delta}^{p q}$, i.e. the amplitude is just the same as in the closed-shell case, with no reference to the spectator. Thus, our Ansatz for $\Psi$ gets modified in this two-body 


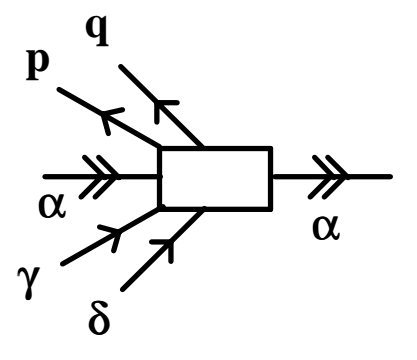

Block III

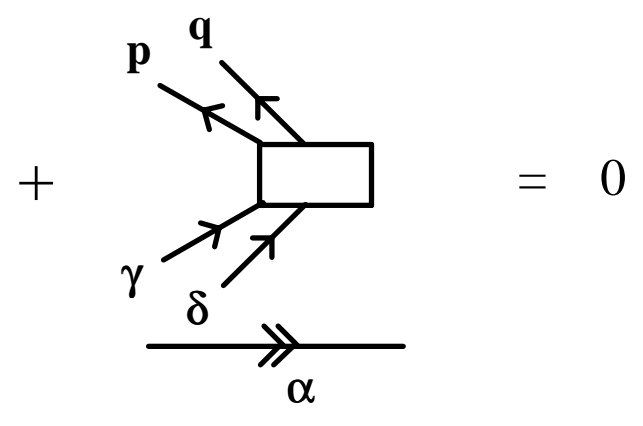

Block IV

Figure 5: Block equation for pseudo-three-body operators: Block III contains the pseudo-threebody operators with spectator scattering, whereas Block IV contains closed-shell two-body operators.

approximation as

$$
\Psi=\left\{\left\{\exp \left(\bar{S}+T_{2}\right)\right\}\right\} \Phi_{\alpha}
$$

with $\bar{S}=\bar{S}_{1}+\bar{S}_{2}$

The idea that one may generate open-shell CC theories for state-energies per se by considering only valence cluster operators in the wave-operator, and by clumping together in the CC equations blocks of different possible ranks but of the same excitations with different number of direct spectator excitations was earlier considered by Mukherjee and Zaitsevskii [30] using a normal ordered wave-operator. The present development uses the more compact and physically more appealing $\{\{\exp (\bar{S})\}\}$ Ansatz to take care of orbital relaxation and correlation effects.

\section{Numerical Applications}

We will discuss here the results obtained by this formalism to generate both the energy of the ground state of the doublet $\mathrm{OH}$ radical, and its electron affinity, using the closed shell $\mathrm{OH}^{-}$as the vacuum. The electron affinity of the radical will be computed as the first IP of the $\mathrm{OH}^{-}$at the radical geometry.

In the VU method the IP is obtained as the difference energy directly, whereas in the VS method the radical state energy itself is calculated. In order for us to be able to compare the relative performance of the VS and the VU methods, the radical state energy in VU method have also been calculated by adding explicitly the anion state energy $E_{0}$ to the IP values. The HartreeFock (HF) orbitals for the $\mathrm{OH}^{-}$ground state at the equilibrium geometry of the $\mathrm{OH}$ radical are used in all the calculations. The equilibrium $\mathrm{OH}$ bond length is 1.83238 a.u. 
The relative success of the two open-shell CC-based theories as compared with the normal ordered version is predicated by their ability to tackle with greater accuracy the orbital relaxation effects associated with the ionization of an electron from the $\mathrm{OH}^{-}$anion, using one and twobody cluster operators. The traditional normal ordered $\{\exp (S)\}$ formalism has also been used to compute the same quantities, to see the effect of orbital relaxations in this method as compared to our current formulation.

We have carried out our VU and VS methods with several basis sets: (i) aug-cc-pVDZ, (ii) aug-cc-pVTZ (without the $f$ functions) and (iii) a different cc-pVTZ (without the $f$ functions) with diffuse $s$ and $p$ functions. We use all the six cartesian components of the $d$ orbitals.

All the doublet state energies and their corresponding IPs, with both the new VS and VU theories, and the corresponding values of the exponential values of the $\{\exp (S)\}$ theories are tabulated in Table 1. The VS version of the $\{\exp (S)\}$ theory is that of Mukherjee and Zaitsevski [30]. Because of the unavailability of the FCI results with these basis sets, comparison with experimental results are done. It is clear that the state energies as well as the EA values with the new formulation include the relaxation as well as the differential correlation effects quite efficiently as compared to those from $\{\exp (S)\}$ in both VS and VU theories.

Table 1: Electron affinity study of $\mathrm{OH}$ radical, using different basis sets

\begin{tabular}{c|c|c|c|c|c|c}
\hline \hline & \multicolumn{2}{|c|}{ VS theory } & \multicolumn{4}{c}{ VU theory } \\
& \multicolumn{2}{|c|}{ State Energies (a.u.) } & \multicolumn{3}{c}{ EA Values (eV) } \\
\hline $\begin{array}{c}\text { Basis } \\
\text { Type }\end{array}$ & $\{\exp (\bar{S})\}$ & $\{\{\exp (\bar{S})\}\}$ & $\{\exp (S)\}$ & $\{\{\exp (S)\}\}$ & Exptl. & $\begin{array}{c}\text { Koopmans' } \\
\text { Value }\end{array}$ \\
\hline I & -75.627858 & -75.621784 & 1.5717 & 1.6540 & $1.83^{a}$ & 2.9101 \\
& & & $(-75.628059)^{b}$ & $(-75.625033)$ & & \\
II & -75.580422 & -75.575777 & 1.6005 & 1.6608 & & 2.9436 \\
& & & $(-75.584965)$ & $(-75.582751)$ & & \\
III & -75.629468 & -75.623728 & 1.7029 & 1.7628 & & 2.9612 \\
& & & $(-75.630625)$ & $(-75.628424)$ & & \\
\hline \hline
\end{tabular}

${ }^{a}[31]^{b}$ The computed state energies are shown in parentheses.

Basis I: cc-pVTZ with diffuse s and p functions

Basis II: aug-cc-pVDZ

Basis III: aug-cc-pVTZ 


\section{Acknowledgements}

One of us (DJ) would like to thank the Council of Scientific and Industrial Research (CSIR), Government of India, New Delhi, for providing him a research fellowship.

\section{References}

1. Cizek, J. Adv. Chem. Phys. 1969, 14, 35.

Cizek, J.; Paldus, J.; Shavitt, I.; Phys. Rev. 1972, A5, 50.

2. Bartlett, R. J. In Modern Electronic Structure Theory; of Advanced Series in Physical Chemistry; Yarkony, D. R., Ed.; World Scientific Publishing: Singapore, 1995; Vol. 2.

3. Crawford, T. D.; Schaefer III, H. F. In Reviews in Computational Chemistry, Lipkowitz, K. B.; Boyd, D. B., Ed.; Wiley-VCH, John Wiley and Sons. Inc.: New York, 2000; Vol. 14.

4. Mukherjee, D.; Moitra, R. K.; Mukhopadhyay, A. Mol. Phys. 1975, 30, 1861.

Mukherjee, D.; Moitra, R. K.; Mukhopadhyay, A. Mol. Phys. 1977, 33, 955.

Mukherjee, D. Pramana, 1979, 12, 203.

5. Haque, A.; Mukherjee, D. J. Chem. Phys. 1984, 80, 5058.

6. Lindgren, I. Int. J. Quant. Chem. 1978, S12, 33.

7. Lindgren, I.; Morrison, J. Atomic Many-Body Theory; Springer Series in Atoms and Plasmas, 1986; Vol. 3.

8. Nooijen, M.; Bartlett, R. J. J. Chem. Phys. 1997, 106, 6441.

9. For extensive surveys of the MRCC methods see, Mukherjee, D.; Pal, S. Adv. Quant. Chem. 1989, 20, 291.

10. Jeziorski, B.; Monkhorst, H. J. Phys. Rev. 1981, A24, 1668.

11. Jeziorski, B.; Paldus, J. J. Chem. Phys. 1988, 88, 5673.

ibid, 1989, 90, 2714.

12. Berkovic, S.; Kaldor, U. Chem. Phys. Lett. 1992, 199, 42.

J. Chem. Phys. 1993, 98, 3090.

13. Rittby, M.; Bartlett, R. J. J. Phys. Chem. 1988, 92, 3033.

14. Scuseria, G. E. Chem. Phys. Lett. 1991, 176, 27.

15. Lee, T. J.; Jayatilaka, D. Chem. Phys. Lett. 1993, 201, 1.

Jayatilaka, D; Lee, T. J. J. Chem. Phys. 1993, 98, 9734.

16. Janssen, C. L.; Schaefer III, H. F. Theo. Chim. Acta. 1991, 79, 1.

17. Li, X.; Paldus, J. J. Chem. Phys. 1994, 101, 8812.

Li, X.; Paldus, J. In Modern Ideas in Coupled Cluster Methods; Ed. Bartlett, R. J., Ed.; World Scientific: Singapore, 1997; Vol. 183. 
18. Jeziorski, B.; Paldus, J.; Jankowski, P. Int. J. Quant. Chem. 1995, 56, 129.

19. Nakatsuji, H.; Hirao, K. J. Chem. Phys. 1978, 68, 2053.

20. Szalay, P. G.; Gauss, J. J. Chem. Phys. 1997, $107,9028$.

21. Nooijen, M.; Bartlett, R. J. J. Chem. Phys. 1996, 104, 2652.

22. Jana, D.; Bandyopadhyay, B.; Mukherjee, D. Theor. Chem. Acc., 1999, 102, 317.

23. Jana, D.; Sinha Mahapatra, U.; Mukherjee, D. Chem. Phys. Lett. 2002, 353, 100.

24. Sinha, D.; Mukhopadhyay, S.; Chaudhuri, R.; Mukherjee, D. Chem. Phys. Lett. 1989, 154, 544.

25. Cederbaum, L. S.; Domcke, W.; Schirmer, J. Phys. Rev. 1980, A22, 206.

26. Mukhopadhyay, D.; Mukherjee, D. Chem. Phys. Lett. 1989, 163, 171. 1991, $17 \%, 441$.

27. Mukhopadhyay, D.; Mukherjee, D. In Applied Many-Body Methods in Spectroscopy and Electronic Structure; Mukherjee, D., Ed.; Plenum Press: New York, 1992; p 261.

28. Mukhopadhyay, D.; Chaudhuri, R.; Mukherjee, D. Chem. Phys. Lett. 1990, 172, 515.

29. Thouless, D. J. Nucl. Phys. 1960, 21, 225.

30. Mukherjee, D.; Zaitsevskii, A. Chem. Phys. Lett. 1995, 233, 605.

31. Celotta, R. J.; Bennett, R. A.; Hall, J. L. J. Chem. Phys. 1974, 60, 1740. 